\title{
Information Geometric Measures of Complexity with Applications to Classical and Quantum Physical Settings
}

\author{
Carlo Cafaro ${ }^{1, *}$ and Sean A. Ali ${ }^{2}$ \\ 1 SUNY Polytechnic Institute, Albany, NY 12203, USA \\ 2 Albany College of Pharmacy and Health Sciences, Albany, NY 12208, USA; sean.ali@acphs.edu \\ * Correspondence: CCafaro@sunypoly.edu
}

check for updates

Citation: Cafaro, C.; Ali, S.A. Information Geometric Measures of Complexity with Applications to Classical and Quantum Physical Settings . Foundations 2021, 1, 45-62. https://doi.org/10.3390/ foundations1010006

Academic Editor: Eugene Oks

Received: 20 July 2021

Accepted: 25 August 2021

Published: 31 August 2021

Publisher's Note: MDPI stays neutral with regard to jurisdictional claims in published maps and institutional affiliations.

Copyright: (c) 2021 by the authors. Licensee MDPI, Basel, Switzerland. This article is an open access article distributed under the terms and conditions of the Creative Commons Attribution (CC BY) license (https:// creativecommons.org/licenses/by/ $4.0 /)$.
Abstract: We discuss the fundamental theoretical framework together with numerous results obtained by the authors and colleagues over an extended period of investigation on the Information Geometric Approach to Chaos (IGAC).

Keywords: information geometry; complexity; classical and quantum physics

PACS: chaos (05.45.-a); complexity (89.70.Eg); entropy (89.70.Cf); inference methods (02.50.Tt); information theory $(89.70 .+\mathrm{c})$; probability theory $(02.50 . \mathrm{Cw})$; riemannian geometry $(02.40 . \mathrm{Ky})$

\section{Theoretical Background}

Statistical models are employed to formulate probabilistic descriptions of systems of arbitrary nature when only partial knowledge about the system is available. Indeed, in recent years, methods of entropic inference [1] have been utilized in conjunction with information geometry (IG) [2] for the purpose of developing complexity indicators of statistical models. From the perspective of this hybrid framework, such complexity indicators can be understood as being quantitative measures that describe the complication of inferring macroscopic predictions about statistical models. In this context, the difficulty of making macroscopic predictions is attributed to the fact that statistical models intrinsically reflect only partial information about the microscopic degrees of freedom of the system being modeled. Initial theoretical investigation in this direction, quoted as the Information Geometric Approach to Chaos (IGAC), was originally proposed by Cafaro in his physics Ph.D. doctoral dissertation in [3].

A general summary of the IGAC framework is described as follows [4,5]: upon identifying the microscopic degrees of freedom of a complex system, one must obtain data and choose important information constraints on the system. Entropic methods are then utilized to obtain an initial, static statistical model of the system. In this way, the system is described by a statistical model specified in terms of probability distributions that are characterized by statistical macrovariables. The statistical macrovariables are determined by the data and the specific functional expression of the information constraints used to implement statistical inferences. The next step in the theoretical scheme is concerned with the temporal evolution of the system. If it is assumed that the system changes, then the corresponding statistical model evolves from its initial to final configurations in a manner specified by Entropic Dynamics (ED, [6]). The ED framework can be viewed as a form of constrained information dynamics that is formulated on statistical manifolds, the elements of which are probability distributions. These distributions, in turn, are in one-to-one relation with an appropriate set of statistical macrovariables that determine a parameter space, where the latter serves to provide a suitable parameterization of points on the original statistical manifold.

Within the context of ED, the change of probability distributions is described in terms of a principle of entropic inference. Specifically, beginning with a known initial 
configuration, change toward the final configuration happens by the maximization of the logarithmic relative entropy (known as the Maximum relative Entropy method-or MrE method in brief, [1]) between any two successive intermediate configurations. We emphasize that ED specifies the expected rather than the actual dynamical paths of the system. Inferences within the ED framework depends on the data and functional form of the selected information constraints used in the MrE algorithm. Indeed, modeling strategies of this kind can only be corroborated a posteriori. This fact implies that in the event inferred predictions fail to match experimental measurements, a new set of information constraints should be chosen. This feature of the MrE algorithm is of critical significance and was recently re-examined by Cafaro and Ali in [7].

The change of probability distributions characterized by the maximization algorithm outlined above prescribes a geodesic evolution for the statistical macrovariables [1]. The Fisher-Rao information metric [2] yields a measure of distance between any two dissimilar probability distributions on a statistical manifold. The notion of distance between elements of a statistical manifold can be regarded as the degree of distinguishability between any two different probability distribution functions. Once the information metric has been obtained, differential geometric techniques can be readily applied to study the geometry of the curved statistical manifold. Broadly speaking, standard Riemannian geometric quantities, such as Christoffel connection coefficients of the second kind, Riemannian curvature tensor, Ricci tensor, Ricci scalar curvature, Weyl anisotropy tensor, sectional curvatures, Killing fields and Jacobi fields (including the IG analogue of Lyapunov exponents) can be calculated in the usual fashion. In particular, the chaoticity (i.e., temporal complexity) of such statistical models can be analyzed via appropriately selected indicators, such as the signs of the Ricci scalar and sectional curvatures of the statistical manifold, non-vanishing Weyl anisotropy tensor, the asymptotic temporal behavior of Jacobi fields and the existence of Killing vectors. Along with the various indicators mentioned above, the notion of complexity within the IGAC framework can also be characterized by the Information Geometric Entropy (IGE), originally proposed in [3]. We make reference to the Ali-Cafaro effort in [8] for a more extensive summary of the IGAC framework that incorporates a set of remarks on entropic evolution and the $\mathrm{MrE}$ algorithm. For a presentation of alternative information geometric descriptions of complexity, we suggest the investigation by Felice, Cafaro and Mancini in [5]. While we certainly appreciate the power of the synthetic, non-component approach to tensor analysis commonly used in theoretical physics (for instance, see [9]), we have nevertheless chosen to employ the component approach in the present paper. In our opinion, the applied nature of our works can be formulated and analyzed more efficiently (and transparently) within the component approach to tensor calculus.

In the next section, we introduce suitable indicators of complexity within the IGAC.

\section{Indicators of Complexity}

In this section, we introduce three indicators of complexity within the context of the IGAC framework. We present the IGE, the curvature of the statistical manifold, and finally, the notion of Jacobi fields arising from the equation of geodesic deviation.

\subsection{Information Geometric Entropy}

We begin this subsection by discussing the IGE. Assuming the elements $\{p(x ; \theta)\}$ of an $n$-dimensional statistical manifold $\mathcal{M}_{s}$ are parameterized by $n$, real-valued variables $\left(\theta^{1}, \ldots, \theta^{n}\right)$, the statistical manifold is defined by the set

$$
\mathcal{M}_{s} \stackrel{\text { def }}{=}\left\{p(x ; \theta): \theta=\left(\theta^{1}, \ldots, \theta^{n}\right) \in \mathcal{D}_{\boldsymbol{\theta}}^{(\text {tot })}\right\} .
$$


We point out that the quantity $x$ in Equation (1) denotes microvariables belonging to the microspace $\mathcal{X}$, whereas the macrovariables $\theta$ appearing in Equation (1) are elements of the parameter space $\mathcal{D}_{\boldsymbol{\theta}}^{(\text {tot })}$ defined by

$$
\mathcal{D}_{\theta}^{\text {(tot) }} \stackrel{\text { def }}{=} \bigotimes_{j=1}^{n} \mathcal{I}_{\theta^{j}}=\left(\mathcal{I}_{\theta^{1}} \otimes \mathcal{I}_{\theta^{2}} \ldots \otimes \mathcal{I}_{\theta^{n}}\right) \subseteq \mathbb{R}^{n} .
$$

The quantity $\mathcal{I}_{\theta j}$ with $1 \leq j \leq n$ in Equation (2) is a subset of $\mathbb{R}^{n}$ that denotes the full range of admissible values of the statistical macrovariables $\theta^{j}$. The IGE serves as an indicator of temporal complexity associated with geodesic paths in the IGAC framework. The IGE is given by

$$
\mathcal{S}_{\mathcal{M}_{s}}(\tau) \stackrel{\text { def }}{=} \log \widetilde{\operatorname{vol}}\left[\mathcal{D}_{\boldsymbol{\theta}}(\tau)\right],
$$

where the average dynamical statistical volume $\widetilde{\operatorname{vol}}\left[\mathcal{D}_{\theta}(\tau)\right]$ is defined as

$$
\widetilde{\operatorname{vol}}\left[\mathcal{D}_{\theta}(\tau)\right] \stackrel{\text { def }}{=} \frac{1}{\tau} \int_{0}^{\tau} \operatorname{vol}\left[\mathcal{D}_{\theta}\left(\tau^{\prime}\right)\right] d \tau^{\prime}
$$

We remark that the temporal averaging operation is denoted by the tilde symbol in Equation (4). Furthermore, the volume $\operatorname{vol}\left[\mathcal{D}_{\boldsymbol{\theta}}\left(\tau^{\prime}\right)\right]$ appearing on the RHS of Equation (4) is given by

$$
\operatorname{vol}\left[\mathcal{D}_{\boldsymbol{\theta}}\left(\tau^{\prime}\right)\right] \stackrel{\text { def }}{=} \int_{\mathcal{D}_{\theta}\left(\tau^{\prime}\right)} \rho\left(\theta^{1}, \ldots, \theta^{n}\right) d^{n} \theta,
$$

where $\rho\left(\theta^{1}, \ldots, \theta^{n}\right)$ is known as the Fisher density and is equal to the square root of the determinant $g(\theta)$ of the Fisher-Rao information metric tensor $g_{\mu v}(\theta)$,

$$
\rho\left(\theta^{1}, \ldots, \theta^{n}\right) \stackrel{\text { def }}{=} \sqrt{g(\theta)} .
$$

The Fisher-Rao information metric tensor $g_{\mu v}(\theta)$ is defined as

$$
g_{\mu \nu}(\theta) \stackrel{\text { def }}{=} \int p(x \mid \theta) \partial_{\mu} \log p(x \mid \theta) \partial_{\nu} \log p(x \mid \theta) d x,
$$

where $\mu, v=1, \ldots, n$ for an $n$-dimensional manifold and $\partial_{\mu} \stackrel{\text { def }}{=} \frac{\partial}{\partial \theta \|^{\mu}}$. The volume $\operatorname{vol}\left[\mathcal{D}_{\boldsymbol{\theta}}\left(\tau^{\prime}\right)\right]$ in Equation (5) can be recast in a more crystalline manner for cases involving statistical manifolds whose information metric tensor has a determinant that can be expressed in a factorized form as follows,

$$
g(\theta)=g\left(\theta^{1}, \ldots, \theta^{n}\right)=\prod_{j=1}^{n} g_{j}\left(\theta^{j}\right)
$$

By using the factorized form of the determinant, the IGE appearing in Equation (3) can be expressed as

$$
\mathcal{S}_{\mathcal{M}_{s}}(\tau)=\log \left\{\frac{1}{\tau} \int_{0}^{\tau}\left[\prod_{j=1}^{n}\left(\int_{\tau_{0}}^{\tau_{0}+\tau^{\prime}} \sqrt{g_{j}\left[\theta^{j}(\xi)\right]} \frac{d \theta^{j}}{d \xi} d \xi\right)\right] d \tau^{\prime}\right\} .
$$

Within the IGAC framework, the leading asymptotic behavior of $\mathcal{S}_{\mathcal{M}_{s}}(\tau)$ in Equation (9) is employed to specify the complexity of the statistical model under investigation. Therefore, it is quite instructive to take into consideration the quantity

$$
\mathcal{S}_{\mathcal{M}_{s}}^{\text {(asymptotic) }}(\tau) \approx \lim _{\tau \rightarrow \infty}\left[\mathcal{S}_{\mathcal{M}_{s}}(\tau)\right]
$$


that is, the leading asymptotic term in the expression of the IGE. The integration space $\mathcal{D}_{\theta}\left(\tau^{\prime}\right)$ in Equation (5) is defined by

$$
\mathcal{D}_{\boldsymbol{\theta}}\left(\tau^{\prime}\right) \stackrel{\text { def }}{=}\left\{\theta: \theta^{j}\left(\tau_{0}\right) \leq \theta^{j} \leq \theta^{j}\left(\tau_{0}+\tau^{\prime}\right)\right\},
$$

where $\theta^{j}=\theta^{j}(\xi)$ with $\tau_{0} \leq \xi \leq \tau_{0}+\tau^{\prime}$ and $\tau_{0}$ denotes the initial value of the affine parameter $\xi$ such that

$$
\frac{d^{2} \theta^{j}(\tilde{\zeta})}{d \xi^{2}}+\Gamma_{i k}^{j} \frac{d \theta^{i}}{d \xi} \frac{d \theta^{k}}{d \xi}=0
$$

The domain of integration $\mathcal{D}_{\boldsymbol{\theta}}\left(\tau^{\prime}\right)$ in Equation (11) is an $n$-dimensional subspace of $\mathcal{D}_{\boldsymbol{\theta}}^{\text {(tot) }}$. The elements of $\mathcal{D}_{\theta}^{\text {(tot) }}$ are $n$-dimensional macrovariables $\{\theta\}$ whose components $\theta^{j}$ are bounded by the limits of integration $\theta^{j}\left(\tau_{0}\right)$ and $\theta^{j}\left(\tau_{0}+\tau^{\prime}\right)$. The temporal functional form of such limits is determined by the integration of the geodesic equations in Equation (12).

The IGE evaluated at a particular instant is specified by the logarithm of the volume of the effective parameter space probed by the system at that instant. In order to coarse-grain the possibly very complex details of the entropic dynamical characterization of the system, however, the process of temporal averaging has been employed. Moreover, in order to remove the effects of potential transient features that may enter the calculation of the expected value of the volume of the effective parameter space, only its asymptotic temporal behavior is taken into consideration. For these reasons, it is evident that the IGE serves as an asymptotic, coarse-grained complexity indicator of dynamical systems in the presence of partial information. For additional specifics concerning the IGE, we refer the interested reader to $[8,10]$.

As a conclusive side remark, we emphasize that it would be interesting to characterize the tendency to increase of the entropy of a physical system that approaches equilibrium as specified by the Boltzmann $\mathbf{H}$ theorem and the second law of thermodynamics [11] from an information geometric perspective. For a recent information geometric interpretation of the entropy production, we refer to [12]. In particular, to understand the possible link between the IGE and the Boltzmann-Shannon entropy, it would be important to study the Kaniadakis $\mathcal{S}_{\mathcal{K}}$ entropy (with $\kappa$ being the so-called deformation parameter) and comprehend how the statistical mechanics based on $\mathcal{S}_{\kappa}$ can be regarded as a natural generalization of the equilibrium Boltzmann-Gibbs statistical mechanics [13]. We leave these intriguing lines of investigations to future efforts.

\subsection{Curvature}

We present here the notion of curvature of statistical manifolds. We begin by recalling that an $n$-dimensional, $\mathbb{C}^{\infty}$ differentiable manifold is defined by a set of points $\mathcal{M}$ endowed with coordinate systems $\mathcal{C}_{\mathcal{M}}$ fulfilling the following two requirements: (1) each element $c \in \mathcal{C}_{\mathcal{M}}$ is a one-to-one mapping from $\mathcal{M}$ to an open subset of $\mathbb{R}^{n} ;$ (2) given any one-to-one mapping $\eta: \mathcal{M} \rightarrow \mathbb{R}^{n}$, we have that $\forall c \in \mathcal{C}_{\mathcal{M}}, \eta \in \mathcal{C}_{\mathcal{M}} \Leftrightarrow \eta \circ c^{-1}$ is a $\mathbb{C}^{\infty}$ diffeomorphism.

In this paper, we focus on Riemannian manifolds $(\mathcal{M}, g)$ where the points of $\mathcal{M}$ are probability distribution functions. It is worth noting that the manifold structure of $\mathcal{M}$ is insufficient to specify in a unique manner the Riemannian metric $g$. On a formal level, an infinite number of Riemannian metrics can be defined on the manifold $\mathcal{M}$. In the context of information geometry however, the selection of the Fisher-Rao information metric (see Equation (7)) as the metric underlying the Riemannian geometry of probability distributions $[2,14,15]$ serves as a primary working assumption. The characterization theorem attributed to Cencov [16] gives significant support for this particular choice of metric. In this characterization theorem, Cencov demonstrates that, up to any arbitrary constant scale factor, the information metric is the only Riemannian metric that is invariant under congruent embeddings (that is, under a family of probabilistically meaningful mappings) of the Markov morphism [16,17]. 
Upon introducing the Fisher-Rao information metric $g_{\mu v}(\theta)$ in Equation (7), standard differential geometric techniques can be used on the space of probability distributions to describe the geometry of the statistical manifold $\mathcal{M}_{s}$. The Ricci scalar curvature $\mathcal{R}_{\mathcal{M}_{s}}$ is one example of such a geometric property, where $\mathcal{R}_{\mathcal{M}_{s}}$ is defined as [18]

$$
\mathcal{R}_{\mathcal{M}_{s}} \stackrel{\text { def }}{=} g^{\mu v} \mathcal{R}_{\mu v}
$$

where $g^{\mu v} g_{v \rho}=\delta_{\rho}^{\mu}$ and $g^{\mu v}=\left(g_{\mu v}\right)^{-1}$. The Ricci tensor $\mathcal{R}_{\mu v}$ appearing in Equation (13) is given as [18]

$$
\mathcal{R}_{\mu \nu} \stackrel{\text { def }}{=} \partial_{\gamma} \Gamma_{\mu \nu}^{\gamma}-\partial_{\nu} \Gamma_{\mu \lambda}^{\lambda}+\Gamma_{\mu \nu}^{\gamma} \Gamma_{\gamma \eta}^{\eta}-\Gamma_{\mu \gamma}^{\eta} \Gamma_{\nu \eta}^{\gamma} .
$$

The Christoffel connection coefficients $\Gamma_{\mu \nu}^{\rho}$ of the second kind that specify the Ricci tensor in Equation (14) are [18]

$$
\Gamma_{\mu \nu}^{\rho} \stackrel{\text { def }}{=} \frac{1}{2} g^{\rho \sigma}\left(\partial_{\mu} g_{\sigma \nu}+\partial_{\nu} g_{\mu \sigma}-\partial_{\sigma} g_{\mu \nu}\right)
$$

Next we consider geodesic curves on statistical manifolds. A geodesic on an ndimensional statistical manifold $\mathcal{M}_{s}$ can be interpreted as the maximum probability trajectory explored by a complex system during its change from an initial $\theta_{\text {initial }}$ to final macrostates $\theta_{\text {final }}$, respectively. Each point along a geodesic path corresponds to a macrostate specified by the macroscopic variables $\theta=\left(\theta^{1}, \ldots, \theta^{n}\right)$. In the context of ED, each component $\theta^{j}$ with $j=1, \ldots, n$ is a solution of the geodesic equation [6],

$$
\frac{d^{2} \theta^{k}}{d \xi^{2}}+\Gamma_{l m}^{k} \frac{d \theta^{l}}{d \xi} \frac{d \theta^{m}}{d \xi}=0 .
$$

At this juncture, we reiterate the fact that each macrostate $\theta$ is in one-to-one correspondence with the probability distribution $p(x \mid \theta)$, with the latter characterizing a distribution of the microstates $x$. It is useful to recognize that the scalar curvature $\mathcal{R}_{\mathcal{M}_{s}}$ can be readily recast as the sum of sectional curvatures $\mathcal{K}\left(e_{\rho}, e_{\sigma}\right)$ of all tangent space planes $T_{p} \mathcal{M}_{s}$ with $p \in \mathcal{M}_{s}$ spanned by pairs of orthonormal basis vectors $\left\{e_{\rho}=\partial_{\theta_{\rho}(p)}\right\}$,

$$
\mathcal{R}_{\mathcal{M}_{s}} \stackrel{\text { def }}{=} \mathcal{R}_{\alpha}^{\alpha} \stackrel{\text { def }}{=} \sum_{\rho \neq \sigma} \mathcal{K}\left(e_{\rho}, e_{\sigma}\right),
$$

where $\mathcal{K}(a, b)$ is given by [18]

$$
\mathcal{K}(a, b) \stackrel{\text { def }}{=} \frac{\mathcal{R}_{\mu v \rho \sigma} a^{\mu} b^{v} a^{\rho} b^{\sigma}}{\left(g_{\mu \sigma} g_{v \rho}-g_{\mu \rho} g_{v \sigma}\right) a^{\mu} b^{v} a^{\rho} b^{\sigma}},
$$

with

$$
a \stackrel{\text { def }}{=} \sum_{\rho}\left\langle a, e^{\rho}\right\rangle e_{\rho}, b \stackrel{\text { def }}{=} \sum_{\rho}\left\langle b, e^{\rho}\right\rangle e_{\rho}, \text { and }\left\langle e_{\rho}, e^{\sigma}\right\rangle \stackrel{\text { def }}{=} \delta_{\rho}^{\sigma} .
$$

We observe that the Riemann curvature tensor $\mathcal{R}_{\alpha \beta \rho \sigma}[18]$ is fully determined by the sectional curvatures $\mathcal{K}\left(e_{\rho}, e_{\sigma}\right)$ where

$$
\mathcal{R}_{\beta \rho \sigma}^{\alpha} \stackrel{\text { def }}{=} g^{\alpha \gamma} \mathcal{R}_{\gamma \beta \rho \sigma} \stackrel{\text { def }}{=} \partial_{\sigma} \Gamma_{\beta \rho}^{\alpha}-\partial_{\rho} \Gamma_{\beta \sigma}^{\alpha}+\Gamma_{\lambda \sigma}^{\alpha} \Gamma_{\beta \rho}^{\lambda}-\Gamma_{\lambda \rho}^{\alpha} \Gamma_{\beta \sigma}^{\lambda} .
$$

The negativity of the Ricci scalar curvature $\mathcal{R}_{\mathcal{M}_{s}}$ is a strong (i.e., a sufficient but not necessary) criterion of local dynamical instability. Moreover, the compactness of the manifold $\mathcal{M}_{S}$ is required to specify genuine chaotic (that is, temporally complex) dynamical systems. In particular, it is evident from Equation (17) that the negativity of $\mathcal{R}_{\mathcal{M}_{s}}$ implies that negative principal curvatures (i.e., extrema of sectional curvatures) are more dominant than positive ones. For this reason, the negativity of $\mathcal{R}_{\mathcal{M}_{s}}$ is a sufficient but not necessary 
requirement for local instability of geodesic flows on statistical manifolds. It is worth mentioning the possible circumstance of scenarios in which negative sectional curvatures are present, but the positive curvatures dominate in the sum of Equation (17) such that $\mathcal{R}_{\mathcal{M}_{s}}$ is a non-negative quantity despite flow instability in those directions. For additional mathematical considerations related to the concept of curvature in differential geometry, we refer to [19].

\subsection{Jacobi Fields}

We introduce here the concept of the Jacobi vector field. It is worth noting that the analysis of stability/instability arising in natural (geodesic) evolutions is readily accomplished by means of the Jacobi-Levi-Civita (JLC) equation for geodesic deviation. This equation is familiar in both theoretical physics (for example, in the case of General Relativity) as well as in Riemannian geometry. The JLC equation describes in a covariant manner, the degree to which neighboring geodesics locally scatter. In particular, the JLC equation effectively connects the curvature properties of an underlying manifold to the stability/instability of the geodesic flow induced thereupon. Indeed, the JLC equation provides a window into a diverse and mostly unexplored field of study concerning the connections among topology, geometry and geodesic instability, and thus to complexity and chaoticity. The use the JLC equation in the setting of information geometry originally appeared in [20].

In what follows, we take into consideration two neighboring geodesic paths $\theta^{\alpha}(\xi)$ and $\theta^{\alpha}(\xi)+\delta \theta^{\alpha}(\xi)$, where the quantity $\xi$ denotes an affine parameter satisfying the geodesic equations,

$$
\frac{d^{2} \theta^{\alpha}}{d \xi^{2}}+\Gamma_{\beta \gamma}^{\alpha}(\theta) \frac{d \theta^{\beta}}{d \xi} \frac{d \theta^{\gamma}}{d \xi}=0,
$$

and

$$
\frac{d^{2}\left[\theta^{\alpha}+\delta \theta^{\alpha}\right]}{d \xi^{2}}+\Gamma_{\beta \gamma}^{\alpha}(\theta+\delta \theta) \frac{d\left[\theta^{\beta}+\delta \theta^{\beta}\right]}{d \xi} \frac{d\left[\theta^{\gamma}+\delta \theta^{\gamma}\right]}{d \xi}=0,
$$

respectively. Noting that to first order in $\delta \theta^{\alpha}$,

$$
\Gamma_{\beta \gamma}^{\alpha}(\theta+\delta \theta) \approx \Gamma_{\beta \gamma}^{\alpha}(\theta)+\partial_{\eta} \Gamma_{\beta \gamma}^{\alpha} \delta \theta^{\eta},
$$

after some algebraic calculations, to first order in $\delta \theta^{\alpha}$, Equation (22) becomes

$$
\frac{d^{2} \theta^{\alpha}}{d \xi^{2}}+\frac{d^{2}\left(\delta \theta^{\alpha}\right)}{d \xi^{2}}+\Gamma_{\beta \gamma}^{\alpha}(\theta) \frac{d \theta^{\beta}}{d \xi} \frac{d \theta^{\gamma}}{d \xi}+2 \Gamma_{\beta \gamma}^{\alpha}(\theta) \frac{d \theta^{\beta}}{d \xi} \frac{d\left(\delta \theta^{\gamma}\right)}{d \xi}+\partial_{\eta} \Gamma_{\beta \gamma}^{\alpha}(\theta) \delta \theta^{\eta} \frac{d \theta^{\beta}}{d \xi} \frac{d \theta^{\gamma}}{d \xi}=0 .
$$

The equation of geodesic deviation can be found by subtracting Equation (21) from Equation (24),

$$
\frac{d^{2}\left(\delta \theta^{\alpha}\right)}{d \xi^{2}}+2 \Gamma_{\beta \gamma}^{\alpha}(\theta) \frac{d \theta^{\beta}}{d \xi} \frac{d\left(\delta \theta^{\gamma}\right)}{d \xi}+\partial_{\eta} \Gamma_{\beta \gamma}^{\alpha}(\theta) \delta \theta^{\eta} \frac{d \theta^{\beta}}{d \xi} \frac{d \theta^{\gamma}}{d \xi}=0 .
$$

Equation (25) can be conveniently recast via the covariant derivatives (see [21], for instance) along the curve $\theta^{\alpha}(\xi)$,

$$
\begin{aligned}
\frac{D^{2}\left(\delta \theta^{\alpha}\right)}{D \xi^{2}} & =\frac{d^{2}\left(\delta \theta^{\alpha}\right)}{d \xi^{2}}+\partial_{\beta} \Gamma_{\rho \sigma}^{\alpha} \frac{d \theta^{\beta}}{d \xi} \delta \theta^{\rho} \frac{d \theta^{\sigma}}{d \xi}+2 \Gamma_{\rho \sigma}^{\alpha} \frac{d\left(\delta \theta^{\rho}\right)}{d \xi} \frac{d \theta^{\sigma}}{d \xi}+ \\
& -\Gamma_{\rho \sigma}^{\alpha} \Gamma_{\kappa \lambda}^{\sigma} \delta \theta^{\rho} \frac{d \theta^{\kappa}}{d \xi} \frac{d \theta^{\lambda}}{d \xi}+\Gamma_{\rho \sigma}^{\alpha} \Gamma_{\kappa \lambda}^{\rho} \delta \theta^{\kappa} \frac{d \theta^{\lambda}}{d \xi} \frac{d \theta^{\sigma}}{d \xi} .
\end{aligned}
$$


The covariant derivative is defined as $D_{\xi} \delta \theta^{\alpha} \stackrel{\text { def }}{=} \partial_{\xi} \delta \theta^{\alpha}+\Gamma_{\xi \kappa}^{\alpha} \delta \theta^{\kappa}$ with $D_{\mathcal{\xi}} \stackrel{\text { def }}{=} D / D \xi$ and $\partial_{\xi} \stackrel{\text { def }}{=} \partial / \partial \xi$, respectively. By combining Equations (25) and (26), and performing some tensor algebra calculations, we obtain

$$
\frac{D^{2}\left(\delta \theta^{\alpha}\right)}{D \xi^{2}}=\left(\partial_{\rho} \Gamma_{\eta \sigma}^{\alpha}-\partial_{\eta} \Gamma_{\rho \sigma}^{\alpha}+\Gamma_{\lambda \sigma}^{\alpha} \Gamma_{\eta \rho}^{\lambda}-\Gamma_{\eta \lambda}^{\alpha} \Gamma_{\rho \sigma}^{\lambda}\right) \delta \theta^{\eta} \frac{d \theta^{\rho}}{d \xi} \frac{d \theta^{\sigma}}{d \xi} .
$$

Finally, the geodesic deviation equation expressed in component form becomes

$$
\frac{D^{2} J^{\alpha}}{D \xi^{2}}+\mathcal{R}_{\rho \eta \sigma}^{\alpha} \frac{d \theta^{\rho}}{d \xi} J^{\eta} \frac{d \theta^{\sigma}}{d \xi}=0
$$

where $J^{\alpha} \stackrel{\text { def }}{=} \delta \theta^{\alpha}$ is the $\alpha$-component of the Jacobi vector field [18]. Equation (28) is known formally as the JLC equation. We observe from the JLC equation in Equation (28) that neighboring geodesics accelerate relative to each other at a rate measured in a direct manner by the Riemannian curvature tensor $R_{\alpha \beta \gamma \delta}$. The quantity $J^{\alpha}$ is defined as,

$$
J^{\alpha}=\delta \theta^{\alpha} \stackrel{\text { def }}{=} \delta_{\phi} \theta^{\alpha}=\left(\frac{\partial \theta^{\alpha}(\xi ; \phi)}{\partial \phi}\right)_{\tau=\text { constant }} \delta \phi,
$$

where $\left\{\theta^{\mu}(\xi ; \phi)\right\}$ denotes the one-parameter $\phi$ family of geodesics whose evolution is specified by means of the affine parameter $\xi$. The Jacobi vector field intensity $J_{\mathcal{M}_{s}}$ on the manifold $\mathcal{M}_{s}$ is given by

$$
J_{\mathcal{M}_{s}} \stackrel{\text { def }}{=}\left(J^{\alpha} g_{\alpha \beta} J^{\beta}\right)^{1 / 2} .
$$

In general, the JLC equation is intractable even at low dimensions. However, in the case of isotropic manifolds, it reduces to

$$
\frac{D^{2} J^{\mu}}{D \xi^{2}}+\mathcal{K} J^{\mu}=0
$$

The sectional curvature $\mathcal{K}$ in Equation (31) assumes a constant value throughout the manifold. In particular, when $\mathcal{K}<0$, unstable solutions of Equation (31) become

$$
J^{\mu}(\xi)=\frac{\omega_{0}^{\mu}}{\sqrt{-\mathcal{K}}} \sinh (\sqrt{-\mathcal{K}} \xi)
$$

with initial conditions $J^{\mu}(0)=0$ and $\frac{d J^{\mu}(0)}{d \tau^{\tau}}=\omega^{\mu}(0)=\omega_{0}^{\mu} \neq 0$, respectively, for any $1 \leq \mu \leq n$ with $n$ being the dimensionality of the underlying manifold. For additional remarks concerning the JLC equation, we refer to $[18,21,22]$.

We point out that it would be intriguing to understand the behavior of the Jacobi vector fields within the geometry of the Kaniadakis statistical mechanics emerging from a one deformation parameter $\kappa$ [13]. We leave this fascinating line of study to future scientific inquiry. For a schematic description of the behavior of the IGE and the Jacobi field for two-dimensional surfaces with distinct (Gaussian) curvatures, we refer to Table 1 and Figure 1.

In the next section, making use of the complexity quantifiers introduced in Equations (3), (17), and (30), we present numerous illustrative examples within the IGAC framework. 
Table 1. Schematic description of the behavior of the IGE and the Jacobi field for different types of two-dimensional surfaces characterized by distinct constant values of their Gaussian curvature. For such surfaces, the sectional and the scalar curvatures coincide, while the Gaussian curvature is simply one-half of the scalar curvature. In particular, positive curvature causes geodesics to converge while negative curvature causes geodesics to spread out. More specifically, in flat, positively, and negatively curved manifolds, the geodesic deviation equation yields deviations of nearby geodesics that exhibit linear, oscillatory, and exponential behaviors, respectively. Moreover, the volumes of the manifolds regions explored during the entropic motion tend to increase while transitioning from positively to negatively curved manifolds. Correspondingly, the IGE exhibits its maximum growth (that is, linear growth) in the presence of exponential instability on negatively curved manifolds.

\begin{tabular}{cccc}
\hline Surface & Curvature & Jacobi Field Behavior & IGE Behavior \\
\hline sphere & positive & oscillatory & sublogarithmic \\
cylinder & zero & linear & logarithmic \\
hyperboloid & negative & exponential & linear \\
\hline
\end{tabular}

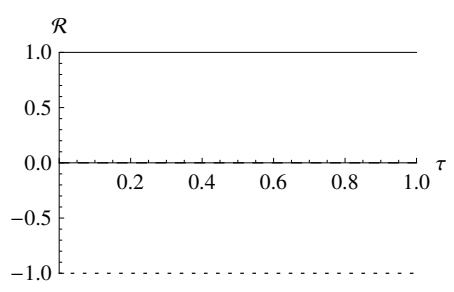

(a)

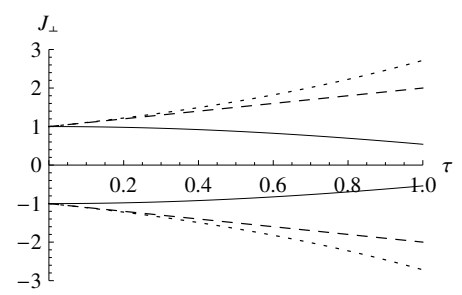

(b)

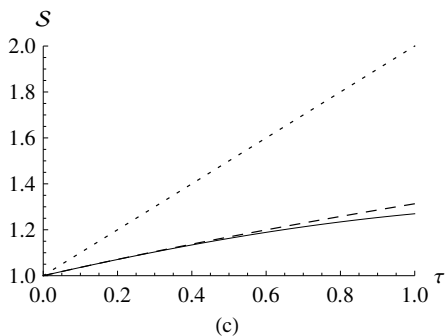

(c)

Figure 1. Graphical depictions of the links among curvature, Jacobi fields, and IGE. In (a), we depict the constant scalar curvature of a positively curved manifold (solid line), a flat manifold (dashed line), and a negatively curved manifold (dotted line). In (b), we illustrate the behavior of the normal components of the Jacobi fields quantifying how nearby geodesics are changing in the normal direction (that is, the direction that is orthogonal to the unit tangent vector of the geodesic) as we move along the geodesics. In the positive, flat, and negative curvature cases, we observe oscillatory behavior (solid line), linear behavior (dashed line), and exponential behavior (dotted line), respectively. Finally, in (c), we plot the temporal behavior of the IGE in the positive (sublogarithmic behavior, solid line), flat (logarithmic behavior, dashed line), and negative (linear behavior, dotted line) curvature cases.

\section{Applications}

In this section, with the help of the three complexity quantifiers introduced above, we report the results of several applications of the IGAC in which the complexity of geodesic trajectories on statistical manifolds are quantified. We present these illustrative examples in a chronological order, from the first one to the last one. For brevity, we omit technical details and confine the presentation to our own information geometric approach to complexity. Early notions and applications of the IGAC originally appeared in [23-25]. For a recent review of the IGAC framework, we refer to $[4,8,26,27]$ and [10], respectively.

\subsection{Uncorrelated Gaussian Statistical Models}

In $[20,28]$, the IGAC framework was employed to study the information geometric features of a system of arbitrary nature, characterized by $l$ degrees of freedom. Each of these degrees of freedom is described by two relevant pieces of information, namely its mean and variance. The infinitesimal line element for this model is given by [28],

$$
d s^{2} \stackrel{\text { def }}{=} \sum_{k=1}^{l} \frac{1}{\sigma_{k}^{2}} d \mu_{k}^{2}+\frac{2}{\sigma_{k}^{2}} d \sigma_{k}^{2}
$$


with $\mu_{k}$ and $\sigma_{k}$ denoting the expectation value and the square root of the variance of the microvariable $x_{k}$, respectively. It was found that the family of statistical models associated to such a system is Gaussian in form. Specifically, it was determined that this set of Gaussian distributions yields a non-maximally symmetric $2 l$-dimensional statistical manifold $\mathcal{M}_{s}$ whose scalar curvature $\mathcal{R}_{\mathcal{M}_{s}}$ assumes a constant negative value that is proportional to the number of degrees of freedom of the system,

$$
\mathcal{R}_{\mathcal{M}_{s}}=-l \text {. }
$$

It was determined that the system explores volume elements on $\mathcal{M}_{s}$ at an exponential rate. In particular, the IGE $\mathcal{S}_{\mathcal{M}_{s}}$ was found to increase in a linear fashion in the asymptotic temporal limit (more precisely, in asymptotic limit of the statistical affine parameter $\tau$ ) and is proportional to the number of degrees of freedom $l$,

$$
\mathcal{S}_{\mathcal{M}_{s}}(\tau) \stackrel{\tau \rightarrow \infty}{\sim} l \lambda \tau
$$

The quantity $\lambda$ in Equation (35) denotes the maximal positive Lyapunov exponent that specifies the statistical model. Geodesic trajectories on $\mathcal{M}_{s}$ were found to be hyperbolic curves. Finally, it was determined that in the asymptotic limit, the Jacobi vector field intensity $J_{\mathcal{M}_{s}}$ is exponentially divergent and is proportional to the number of degrees of freedom $l$,

$$
J_{\mathcal{M}_{s}}(\tau) \stackrel{\tau \rightarrow \infty}{\sim} l \exp (\lambda \tau)
$$

Given that the exponential divergence of the Jacobi vector field intensity $J_{\mathcal{M}_{s}}$ is an established classical feature of chaos, based on the results displayed in Equations (34)-(36), the authors suggest that $\mathcal{R}_{\mathcal{M}_{s}}, \mathcal{S}_{\mathcal{M}_{s}}$ and $J_{\mathcal{M}_{s}}$ each behave as legitimate measures of chaoticity, with each indicator being proportional to the number of Gaussian-distributed microstates of the system. Although this result was verified in the context of this special scenario, the proportionality among $\mathcal{R}_{\mathcal{M}_{s}}, \mathcal{S}_{\mathcal{M}_{s}}$ and $J_{\mathcal{M}_{s}}$ constitutes the first known example appearing in the literature of a possible connection among information geometric indicators of chaoticity obtained from probabilistic modeling of dynamical systems.In this first example, we have compared all three measures $\mathcal{R}_{\mathcal{M}_{s}}, \mathcal{S}_{\mathcal{M}_{s}}$ and $J_{\mathcal{M}_{s}}$. Although we have not performed such a comparative analysis in all applications, we shall attempt to mention curvature and/or Jacobi vector field intensity behaviors whenever possible. Our emphasis here is especially on our entropic measure of complexity. For more details on the other types of complexity indicators, we refer to our original works cited in this manuscript.

\subsection{Correlated Gaussian Statistical Models}

In [29], the IGAC framework was used to analyze the information constrained dynamics of a system comprised of two correlated, Gaussian-distributed microscopic degrees of freedom each having the same variance. The infinitesimal line element for this model is given by [29]

$$
d s^{2} \stackrel{\text { def }}{=} \frac{1}{\sigma^{2}}\left[\frac{1}{1-r^{2}} d \mu_{x}^{2}+\frac{1}{1-r^{2}} d \mu_{y}^{2}-\frac{2 r}{1-r^{2}} d \mu_{x} d \mu_{y}+4 d \sigma^{2}\right],
$$

with $\mu_{x}$ and $\mu_{y}$ denoting the expectation values of the microvariables $x$ and $y$. The quantity $\sigma^{2}$, instead, is the variance while $r$ is the usual correlation coefficient between $x$ and $y$. The scalar curvature $\mathcal{R}_{\mathcal{M}_{s}}$ of the manifold with line element in Equation (37) is $\mathcal{R}_{\mathcal{M}_{s}}=-3 / 2$. The inclusion of microscopic correlations give rise to asymptotic compression of the statistical macrostates explored by the system at a faster rate than that observed in the absence of microscopic correlations. Specifically, it was determined that in the asymptotic limit

$$
\left[\exp \left(\mathcal{S}_{\mathcal{M}_{s}}(\tau)\right)\right]_{\text {correlated }} \stackrel{\tau \rightarrow \infty}{\sim} \mathcal{F}(r) \cdot\left[\exp \left(\mathcal{S}_{\mathcal{M}_{s}}(\tau)\right)\right]_{\text {uncorrelated }^{\prime}}
$$


where the function $\mathcal{F}(r)$ in Equation (38) with $0 \leq \mathcal{F}(r) \leq 1$ is defined as [29]

$$
\mathcal{F}(r) \stackrel{\text { def }}{=} \frac{1}{2^{\frac{5}{2}}}\left[\sqrt{\frac{4\left(4-r^{2}\right)}{\left(2-2 r^{2}\right)^{2}}}\left(\frac{2+r}{4\left(1-r^{2}\right)}\right)^{-\frac{3}{2}}\right] .
$$

The function $\mathcal{F}(r)$ is a monotone decreasing compression envelope $\forall r \in(0,1)$. This result provides an explicit link between correlations at the microscopic level and complexity at the macroscopic level. It also furnishes a transparent and concise description of the functional change of the macroscopic complexity of the underlying statistical manifold caused by the occurrence of microscopic correlations.

\subsection{Inverted Harmonic Oscillators}

Generally speaking, the fundamental issues addressed by the General Theory of Relativity are twofold: firstly, one wishes to understand how the geometry of spacetime evolves in response to the presence of mass-energy distributions; secondly, one seeks to investigate how configurations of mass-energy move in dynamical spacetime geometry. By contrast, within the IGAC framework, one is concerned only with the manner in which systems move within a given statistical geometry, while the evolution of the statistical manifold itself is neglected. The recognition that there exist two separate and distinct characteristics to consider regarding the interplay between mass-energy and spacetime geometry served as a catalyst in the development of the IGAC framework, ultimately leading to a rather interesting finding. The first result obtained in this novel research direction was proposed by Caticha and Cafaro in [30]. In that article, the possibility of utilizing well established principles of inference to obtain Newtonian dynamics from relevant prior information encoded in a suitable statistical manifold was investigated. The primary working assumption in that derivation was the assumed existence of an irreducible uncertainty in the location of particles. This uncertainty requires the state of a particle to be described by a probability distribution. The resulting configuration space is therefore a statistical manifold whose Riemannian geometry is specified by the Fisher-Rao information metric. The expected trajectory is a consequence of the $\mathrm{MrE}$ method, with the latter being regarded as a principle of inference. An unexpected consequence of this approach is that no additional physical postulates such as an equation of motion, principle of least action, nor the concept of momentum, mass, phase space or external time are required. Newton's mechanics involving any number of self-interacting particles as well as particles interacting with external fields is entirely recovered by the resulting entropic dynamics. Indeed, a powerful result of this approach is the fact that interactions among particles as well as particle masses are all justified in terms of the underlying statistical manifold.

Our next example will be of a more applied nature. In [31,32], Zurek and Paz explored the effects of decoherence in quantum chaos by analyzing a single unstable harmonic oscillator with frequency $\Omega$ and potential $V(x)$,

$$
V(x) \stackrel{\text { def }}{=}-\frac{\Omega^{2} x^{2}}{2},
$$

coupled to an external environment. They determined that in the reversible classical limit, the von Neumann entropy of such a system increases linearly at a rate determined by the Lyapunov exponent $\Omega$ according to

$$
\mathcal{S}_{\text {quantum }}^{\text {(chatic) }}(\tau) \stackrel{\tau \rightarrow \infty}{\sim} \Omega \tau .
$$

Building upon the results obtained in [30], an information geometric analogue of the Zurek-Paz quantum chaos criterion in the classical reversible limit was proposed in $[33,34]$. In these works, the IGAC framework was employed to study a set of $l$, three-dimensional, 
anisotropic, uncoupled, inverted harmonic oscillators (IHO) with an Ohmic distributed frequency spectrum.In this example, the infinitesimal line element is given by

$$
d s^{2} \stackrel{\text { def }}{=}[1-\Phi(\theta)] \delta_{\mu v}(\theta) d \theta^{\mu} d \theta^{v},
$$

where $\Phi(\theta)$ is defined as

$$
\Phi(\theta)=\sum_{k=1}^{l} u_{k}(\theta)
$$

with $u_{k}(\theta) \stackrel{\text { def }}{=}-(1 / 2) \omega_{k}^{2} \theta_{k}^{2}$ and $\omega_{k}$ being the frequency of the $k$-th inverted harmonic oscillator. Neglecting mathematical details, it was demonstrated in $[33,34]$ that the asymptotic temporal behavior of the IGE for such a system becomes

$$
\mathcal{S}_{\mathcal{M}_{\mathrm{IHO}}^{(l)}}\left(\tau ; \omega_{1}, \ldots, \omega_{l}\right) \stackrel{\tau \rightarrow \infty}{\sim} \Omega \tau
$$

where,

$$
\Omega \stackrel{\text { def }}{=} \sum_{i=1}^{l} \omega_{i}
$$

and $\omega_{i}$ with $1 \leq i \leq l$ is the frequency of the $i^{\text {th }}$ IHO. Equation (44) indicates an asymptotic, linear IGE growth for the set of IHOs and can be regarded as an extension of the result of Zurek and Paz appearing in [31,32] to an ensemble of anisotropic, uncoupled, inverted harmonic oscillators in the context of the IGAC. We remark that Equation (44) was proposed as the classical IG analogue of Equation (41) in [33,34].

\subsection{Quantum Spin Chains}

In $[35,36]$, the IGAC was used to study the ED on statistical manifolds whose elements are classical probability distribution functions routinely employed in the study of regular and chaotic quantum energy level statistics. Specifically, an IG description of the chaotic (integrable) energy level statistics of a quantum antiferromagnetic Ising spin chain immersed in a tilted (transverse) external magnetic field was presented. The IGAC of a Poisson distribution coupled to an Exponential bath (that specifies a spin chain in a transverse magnetic field and corresponds to the integrable case) along with that of a Wigner-Dyson distribution coupled to a Gaussian bath (that specifies a spin chain in a tilted magnetic field and corresponds to the chaotic case) were investigated. The line elements in the integrable and chaotic cases are given by

$$
d s_{\text {integrable }}^{2} \stackrel{\text { def }}{=} d s_{\text {Poisson }}^{2}+d s_{\text {Exponential }}^{2}=\frac{1}{\mu_{A}^{2}} d \mu_{A}^{2}+\frac{1}{\mu_{B}^{2}} d \mu_{B^{\prime}}^{2}
$$

and,

$$
d s_{\text {chaotic }}^{2} \stackrel{\text { def }}{=} d s_{\text {Wigner-Dyson }}^{2}+d s_{\text {Gaussian }}^{2}=\frac{4}{\mu_{A}^{\prime 2}} d \mu_{A}^{\prime 2}+\frac{1}{\sigma_{B}^{\prime 2}} d \mu_{B}^{\prime 2}+\frac{2}{\sigma_{B}^{\prime 2}} d \sigma_{B}^{\prime 2},
$$

respectively. In Equation (46), $\mu_{A}$ and $\mu_{B}$ are the average spacing of the energy levels and the average intensity of the magnetic field, respectively. A similar notation is employed for the second scenario described in Equation (47) where, clearly, $\sigma_{B}^{\prime 2}$ denotes the variance of the intensity of the magnetic field. Remarkably, it was determined that in the former case, the IGE shows asymptotic logarithmic growth,

$$
\mathcal{S}_{\mathcal{M}_{s}}^{\text {(integrable) }}(\tau) \stackrel{\tau \rightarrow \infty}{\sim} c \log (\tau)+\tilde{c},
$$

whereas in the latter case, the IGE shows asymptotic linear growth,

$$
\mathcal{S}_{\mathcal{M}_{s}}^{\text {(chaotic) }}(\tau) \stackrel{\tau \rightarrow \infty}{\sim} \mathcal{K} \tau
$$


We emphasize that the quantities $c$ and $\tilde{c}$ in Equation (48) are integration constants that depend upon the dimensionality of the statistical manifold and the boundary constraint conditions on the statistical variables, respectively. The quantity $\mathcal{K}$ appearing in Equation (49) denotes a model parameter describing the asymptotic temporal rate of change of the IGE. The findings described above suggest that the IGAC framework may prove useful in the analysis of applications involving quantum energy level statistics. It is worth noting that in such cases, the IGE effectively serves the role of the standard entanglement entropy used in quantum information science [37,38].

\subsection{Statistical Embedding and Complexity Reduction}

Expanding upon the analysis presented in [39], Cafaro and Mancini utilized the IGAC framework in [40] to study the $2 l$-dimensional Gaussian statistical model $\mathcal{M}_{S}$ induced by an appropriate embedding within a larger $4 l$-dimensional Gaussian manifold. The geometry of the $4 l$-dimensional Gaussian manifold is defined by a Fisher-Rao information metric $g_{\mu v}$ with non-vanishing off-diagonal elements. It should be noted that these non-vanishing off-diagonal terms arise due to the occurrence of macroscopic correlation coefficients $\rho_{k}$ with $1 \leq k \leq l$ that specify the embedding constraints among the statistical variables on the larger manifold. The infinitesimal line element is given by [40]

$$
d s^{2} \stackrel{\text { def }}{=} \sum_{k=1}^{l} \frac{1}{\sigma_{2 k-1}^{2}}\left[d \mu_{2 k-1}^{2}+2 \rho_{2 k-1} d \mu_{2 k-1} d \sigma_{2 k-1}+2 d \sigma_{2 k-1}^{2}\right]
$$

with $\rho_{2 k-1}$ defined as

$$
\rho_{2 k-1} \stackrel{\text { def }}{=} \frac{\frac{\partial \mu_{2 k}}{\partial \mu_{2 k-1}} \frac{\partial \mu_{2 k}}{\partial \sigma_{2 k-1}}}{\left[1+\left(\frac{\partial \mu_{2 k}}{\partial \mu_{2 k-1}}\right)^{2}\right]^{1 / 2}\left[2+\frac{1}{2}\left(\frac{\partial \mu_{2 k}}{\partial \sigma_{2 k-1}}\right)^{2}\right]^{1 / 2}},
$$

where $\sigma_{2 k}=\sigma_{2 k-1}$ and $\mu_{2 k}=\mu_{2 k}\left(\mu_{2 k-1}, \sigma_{2 k-1}\right)$ for any $1 \leq k \leq l$. Two significant results were obtained. First, a power law decay of the IGE at a rate determined by the correlation coefficients $\rho_{k}$ was observed

$$
\mathcal{S}_{\mathcal{M}_{s}}\left(\tau ; l, \lambda_{k}, \rho_{k}\right) \stackrel{\tau \rightarrow \infty}{\sim} \log \left[\Lambda\left(\rho_{k}\right)+\frac{\tilde{\Lambda}\left(\rho_{k}, \lambda_{k}\right)}{\tau}\right]^{l},
$$

with $\rho_{k}=\rho_{s} \forall k$ and $s=1, \ldots, l$, where

$$
\begin{gathered}
\Lambda\left(\rho_{k}\right) \stackrel{\text { def }}{=} \frac{2 \rho_{k} \sqrt{2-\rho_{k}^{2}}}{1+\sqrt{\Delta\left(\rho_{k}\right)}}, \tilde{\Lambda}\left(\rho_{k}, \lambda_{k}\right) \stackrel{\text { def }}{=} \frac{\sqrt{\Delta\left(\rho_{k}\right)\left(2-\rho_{k}^{2}\right)} \log \left[\Sigma\left(\rho_{k}, \lambda_{k}, \alpha_{ \pm}\right)\right]}{\rho_{k} \lambda_{k}}, \\
\text { and } \alpha_{ \pm}\left(\rho_{k}\right) \stackrel{\text { def }}{=} \frac{1}{2}\left(3 \pm \sqrt{\Delta\left(\rho_{k}\right)}\right) .
\end{gathered}
$$

The quantity $\Sigma\left(\rho_{k}, \lambda_{k}, \alpha_{ \pm}\right)$is a strictly positive function of its arguments for $0 \leq \rho_{k}<1$ and is given by [40]

$$
\Sigma\left(\rho_{k}, \lambda_{k}, \alpha_{ \pm}\right) \stackrel{\text { def }}{=}-\frac{\Xi_{k}}{4 \lambda_{k}} \frac{1+\sqrt{\Delta\left(\rho_{k}\right)}}{1-\sqrt{\Delta\left(\rho_{k}\right)}} \sqrt{\frac{2 \alpha_{-}\left(\rho_{k}\right)}{\alpha_{+}\left(\rho_{k}\right)}},
$$

where $\Xi_{k}$ and $\lambda_{k}$ are real, positive constants of integration, and

$$
\Delta\left(\rho_{k}\right) \stackrel{\text { def }}{=} 1+4 \rho_{k}^{2} .
$$

Equation (52) represents the first main finding reported in [40] and can be interpreted as a quantitative indication that the IGC of a system decreases in response to the emergence of correlational structures. Second, it was demonstrated that the presence of embedding 
constraints among the Gaussian macrovariables of the larger $4 l$-dimensional manifold results in an attenuation of the asymptotic exponential divergence of the Jacobi field intensity on the embedded $2 l$-dimensional manifold. Neglecting mathematical details, it was determined in [40] that in the asymptotic limit $\tau \gg 1$,

$$
0 \leq \frac{J_{\mathcal{M}_{s}}^{2 l-\text { embedded }}(\tau)}{J_{\mathcal{M}_{s}}^{4 l \text {-larger }}(\tau)}<1
$$

Equation (56) constitutes the second main finding reported in [40]. The observed attenuation of the asymptotic exponential divergence of the Jacobi vector field associated with the larger $4 l$-manifold, suggests that the occurrence of such embedding constraint relations results in an asymptotic compression of the macrostates explored on the statistical manifold $\mathcal{M}_{s}$. These two findings serve to advance, in a non-trivial manner, the goal of developing a description of complexity of either macroscopically or microscopically correlated, multi-dimensional Gaussian statistical models relevant in the modeling of complex systems.

\subsection{Entanglement Induced via Scattering}

Guided by the original study appearing in [41], the IGAC framework was employed to furnish an IG viewpoint on the phenomena of quantum entanglement emerging via $s$-wave scattering between interacting Gaussian wave packets in $[42,43]$. Within the IGAC framework, the pre and post quantum scattering scenarios associated with elastic, headon collision are hypothesized to be macroscopic manifestations arising from underlying microscopic statistical structures. By exploiting this working hypothesis, the pre and post quantum scattering scenarios were modeled by uncorrelated and correlated Gaussian statistical models, respectively.Using the standard notation used so far in this article, the infinitesimal line elements in the absence and presence of correlations are given by

$$
d s_{\text {no-correlations }}^{2}=\frac{1}{\sigma^{2}}\left[d \mu_{x}^{2}+d \mu_{y}^{2}+4 d \sigma^{2}\right]
$$

and,

$$
d s_{\text {correlations }}^{2} \stackrel{\text { def }}{=} \frac{1}{\sigma^{2}}\left[\frac{1}{1-r^{2}} d \mu_{x}^{2}+\frac{1}{1-r^{2}} d \mu_{y}^{2}-\frac{2 r}{1-r^{2}} d \mu_{x} d \mu_{y}+4 d \sigma^{2}\right],
$$

respectively. The scalar curvature $\mathcal{R}_{\mathcal{M}_{S}}$ of the manifolds with line elements in Equations (57) and (58) is $\mathcal{R}_{\mathcal{M}_{s}}=-3 / 2$. Using such a hybrid modeling approach enabled the authors to express the entanglement strength in terms of the scattering potential and incident particle energy. Moreover, the manner in which the entanglement duration is related to the scattering potential and incident particle energy was furnished with a possible explanation. Finally, the link between complexity of informational geodesic paths and entanglement was discussed. In particular, it was demonstrated that in the asymptotic limit,

$$
\left[\exp \left(\mathcal{S}_{\mathcal{M}_{S}}(\tau)\right)\right]_{\text {correlated }} \stackrel{\tau \rightarrow \infty}{\sim} \mathcal{F}(r) \cdot\left[\exp \left(\mathcal{S}_{\mathcal{M}_{s}}(\tau)\right)\right]_{\text {uncorrelated }}
$$

where the function $\mathcal{F}(r)$ in Equation (59) with $0 \leq \mathcal{F}(r) \leq 1$ is defined as

$$
\mathcal{F}(r) \stackrel{\text { def }}{=} \sqrt{\frac{1-r}{1+r}} .
$$

The function $\mathcal{F}(r)$ is a monotone decreasing compression factor with $0<r<1$. The analysis proposed in $[42,43]$ is a significant progress toward the understanding among the concepts of entanglement and statistical micro-correlations, as well as the impact of microcorrelations on the complexity of informational geodesic paths. The finding appearing in 
Equation (59) suggests that the IGAC construct may prove useful in developing a sound IG perspective of the phenomenon of quantum entanglement.

\subsection{Softening of Classical Chaos by Quantization}

Expanding upon the original analysis presented in [44-46], the IGAC framework was utilized to investigate the entropic dynamics and information geometry of a threedimensional Gaussian statistical model as well as the two-dimensional Gaussian statistical model derived from the former model by introducing the following macroscopic information constraint,

$$
\sigma_{x} \sigma_{y}=\Sigma^{2}
$$

where $\Sigma^{2} \in \mathbb{R}_{0}^{+}$. The quantities $x$ and $y$ label the microscopic degrees of freedom of the system. The constraint given by Equation (61) resembles the standard minimum uncertainty relation encountered in quantum mechanics [47]. The infinitesimal line elementsin the $3 D$ and $2 D$-Gaussian statistical models are given by

$$
d s_{3 D}^{2} \stackrel{\text { def }}{=} \frac{1}{\sigma_{x}^{2}} d \mu_{x}^{2}+\frac{2}{\sigma_{x}^{2}} d \sigma_{x}^{2}+\frac{2}{\sigma_{y}^{2}} d \sigma_{y}^{2}
$$

and,

$$
d s_{2 D}^{2} \stackrel{\text { def }}{=} \frac{1}{\sigma^{2}} d \mu_{x}^{2}+\frac{4}{\sigma^{2}} d \sigma^{2}
$$

respectively. Note that the expectation value $\mu_{y}$ of the microvariable $y$ is set equal to zero in Equation (62), while $\sigma_{x}=\sigma$ with $\sigma_{x} \sigma_{y}=\Sigma^{2}$ in Equation (63). Furthermore, the scalar curvatures corresponding to the $3 D$ and $2 D$ cases are equal to $\mathcal{R}_{3 D}=-1$ and $\mathcal{R}_{2 D}=-1 / 2$, respectively. It was determined that the complexity of the $2 D$-Gaussian statistical model specified by the IGE is relaxed when compared with the complexity of the $3 D$-Gaussian statistical model,

$$
\mathcal{S}_{\mathcal{M}_{s}}^{(2 D)}(\tau) \stackrel{\tau \rightarrow \infty}{\sim}\left(\frac{\lambda_{2 D}}{\lambda_{3 D}}\right) \cdot \mathcal{S}_{\mathcal{M}_{s}}^{(3 D)}(\tau)
$$

with $\lambda_{2 D}$ and $\lambda_{3 D}$ being both positive model parameters (satisfying the condition $\lambda_{2 D} \leq$ $\lambda_{3 D}$ ) that express the asymptotic temporal rates of change of the IGE in the $2 D$ and $3 D$ cases, respectively. Motivated by the connection between the macroscopic information constraint (61) on the variances and the phase-space coarse-graining due to the Heisenberg uncertainty relations, the authors suggest their work may shed light on the phenomenon of classical chaos suppression arising from the process of quantization when expressed in an IG setting. It is worth noting that a similar analysis was implemented in [48] where the work in [47] was generalized to a scenario where-in conjunction with the macroscopic constraint in Equation (61) - the microscopic degrees of freedom $x$ and $y$ of the system are also correlated.

\subsection{Topologically Distinct Correlational Structures}

In [49], the asymptotic behavior of the IGE associated with either bivariate or trivariate Gaussian statistical models, with or without micro-correlations, was analyzed by Felice and coworkers. For correlated cases, several correlational configurations among the microscopic degrees of freedom of the system were taken into consideration. It was found that the complexity of macroscopic inferences is dependent on the quantity of accessible microscopic information, as well as on how such microscopic information is correlated. Specifically, in the mildly connected case defined by a trivariate statistical model with two correlations among the three degrees of freedom of the system, the infinitesimal line element is

$$
\left(\left[d s^{2}\right]_{\text {trivariate }}^{\text {(mildly connected) }}\right)_{\text {correlated }} \stackrel{\text { def }}{=} \frac{1}{\sigma^{2}} \frac{3-4 r}{1-2 r^{2}} d \mu^{2}+\frac{6}{\sigma^{2}} d \sigma^{2} .
$$


Moreover, the infinitesimal line element in the uncorrelated trivariate case is given by

$$
\left(\left[d s^{2}\right]_{\text {trivariate }}\right)_{\text {uncorrelated }} \stackrel{\text { def }}{=} \frac{3}{\sigma^{2}} d \mu^{2}+\frac{6}{\sigma^{2}} d \sigma^{2} .
$$

In Equations (65) and (66), $\mu, \sigma$, and $r$ denote the expectation value, the standard deviation, and the correlation coefficient, respectively. It was determined that in the asymptotic limit,

$$
\left(\exp \left[\mathcal{S}_{\text {trivariate }}^{\text {(mildly connected) }}(\tau)\right]\right)_{\text {correlated }} \stackrel{\tau \rightarrow \infty}{\sim} \tilde{\mathcal{R}}_{\text {trivariate }}^{\text {(mildly connected) }}(r)\left(\exp \left[\mathcal{S}_{\text {trivariate }}^{\text {(mildly connected) }}(\tau)\right]\right)_{\text {uncorrelated }^{\prime}}
$$

where

$$
\tilde{\mathcal{R}}_{\text {trivariate }}^{\text {(mildly connected) }}(r) \stackrel{\text { def }}{=} \sqrt{\frac{3\left(1-2 r^{2}\right)}{3-4 r}} .
$$

In Equation (67), the quantity $r$ is the micro-correlation coefficient. The function $\tilde{\mathcal{R}}_{\text {trivariate }}^{\text {(mildly connected) }}(r)$ shows non-monotone behavior in the correlation parameter $r$ and assumes a value of zero at the extrema of the permitted range $r \in(-\sqrt{2} / 2, \sqrt{2} / 2)$. By contrast, for closed bivariate configurations where all microscopic variables are correlated with each other, the complexity ratio between correlated and uncorrelated cases presents monotone behavior in the correlation parameter $r$. For example, in the fully connected bivariate Gaussian case with $\mu_{x}=\mu_{y}=\mu$ and $\sigma_{x}=\sigma_{y}=\sigma$, the infinitesimal line element is

$$
\left(\left[d s^{2}\right]_{\text {bivariate }}^{\text {(fully connected) }}\right)_{\text {correlated }} \stackrel{\text { def }}{=} \frac{2}{\sigma^{2}} \frac{1}{1+r} d \mu^{2}+\frac{4}{\sigma^{2}} d \sigma^{2} .
$$

It was found that

$$
\left(\exp \left[\mathcal{S}_{\text {bivariate }}^{\text {(fully connected) }}(\tau)\right]\right)_{\text {correlated }} \stackrel{\tau \rightarrow \infty}{\sim} \tilde{\mathcal{R}}_{\text {bivariate }}^{\text {ffully connected) }}(r)\left(\exp \left[\mathcal{S}_{\text {bivariate }}^{\text {(fully connected) }}(\tau)\right]\right)_{\text {uncorrelated }^{\prime}}
$$

where

$$
\tilde{\mathcal{R}}_{\text {bivariate }}^{\text {(fully connected) }}(r) \stackrel{\text { def }}{=} \sqrt{1+r} .
$$

Finally, in the fully connected trivariate Gaussian case with trivariate models having all microscopic variables correlated with each other, the infinitesimal line element is

$$
\left(\left[d s^{2}\right]_{\text {trivariate }}^{\text {(fully connected) }}\right)_{\text {correlated }} \stackrel{\text { def }}{=} \frac{3}{\sigma^{2}} \frac{1}{1+2 r} d \mu^{2}+\frac{6}{\sigma^{2}} d \sigma^{2} .
$$

It was determined in this case that

$$
\left(\exp \left[\mathcal{S}_{\text {trivariate }}^{\text {(fully conected) }}(\tau)\right]\right)_{\text {correlated }} \stackrel{\tau \rightarrow \infty}{\sim} \tilde{\mathcal{R}}_{\text {trivariate }}^{\text {(fully connected) }}(r)\left(\exp \left[\mathcal{S}_{\text {trivariate }}^{\text {(fully connected) }}(\tau)\right]\right)_{\text {uncorrelated }^{\prime}}
$$

where

$$
\tilde{\mathcal{R}}_{\text {trivariate }}^{\text {(fully coneded) }}(r) \stackrel{\text { def }}{=} \sqrt{1+2 r} \text {. }
$$

These results imply that in the fully connected bivariate and trivariate configurations, the ratios $\tilde{\mathcal{R}}_{\text {bivariate }}^{\text {(fully coneded) }}(r)$ and $\tilde{\mathcal{R}}_{\text {trivariate }}^{\text {(fully connected) }}(r)$ both present monotone behavior in $r$ over the open intervals $(-1,1)$ and $(-1 / 2,1)$, respectively. On the other hand, in the mildly connected trivariate scenario appearing in Equation (67), an extremum in the function $\tilde{\mathcal{R}}_{\text {trivariate }}^{\text {(mildly conected) }}(r)$ occurs at $r_{\text {peak }}=1 / 2 \geq 0$. Such a distinctly different behavior between mildly and fully connected trivariate configurations can be attributed to the fact that when making statistical inferences subject to the hypothesis of three positively correlated Gaussian random variables, the system becomes frustrated because the maximum entropy favorable state - characterized by minimum complexity - is incompatible with the initial working hypothesis. Guided by these results, it was suggested in [49] that the impossibility of realizing the maximally favorable state for specific correlational configurations among microscopic degrees of freedom, viewed from an entropic inference perspective, yields an 
information geometric analogue of the statistical physics frustration effect that arises when loops are present [50].

\section{Final Remarks}

In this paper, we discussed the primary results obtained by the authors and colleagues over an extended period of work on the IGAC framework. A summary of the IGAC applications can be found in Table 2. For ease of readability, we have chosen to omit technicalities in our discussion. We are aware of several unresolved issues within the IGAC framework, including a deep understanding of the foundational aspects of the IGE measure of complexity. Further developments of the framework are necessary, especially within a fully quantum mechanical setting. For a more detailed list on limitations and future directions of the IGAC approach, we refer the interested reader to [8]. In particular, we mentioned there that one of our main objectives in the near future is to extend our comprehension of the relationship between the IGE and the Kolmogorov-Sinai dynamical entropy [51], the coarse-grained Boltzmann entropy [51] and the von Neumann entropy [52], depending upon the peculiarity of the system being investigated. Despite its limitations, we are pleased that our theoretical modeling approach is steadily gaining interest in the community of researchers. Indeed, there appears to be an increasing number of scientists who either actively use, or who's work is linked to the theoretical framework described in the present brief feature review article [53-78].

Table 2. Schematic description of existing mathematical, classical, and quantum investigations within the IGAC.

\begin{tabular}{ccc}
\hline Math \& IGAC & Classical \& IGAC & Quantum \& IGAC \\
\hline Micro and macro correlations & Geometrization of Newtonian mechanics Spin chains and energy levels statistics \\
Statistical embeddings & Inverted harmonic oscillators & Scattering induced entanglement \\
Topology and correlational structures & Macro effects from micro information & Softening chaoticity by quantization \\
\hline
\end{tabular}

Author Contributions: The authors contributed equally to this work. Both authors have read and agreed to the published version of the manuscript.

Funding: This research received no external funding.

Institutional Review Board Statement: Not applicable.

Informed Consent Statement: Not applicable.

Data Availability Statement: No new data were created or analyzed in this study. Data sharing is not applicable to this article.

Acknowledgments: C. C. acknowledges the hospitality of the United States Air Force Research Laboratory in Rome where part of his initial contribution to this work was completed.

Conflicts of Interest: The authors declare no conflict of interest.

\section{References}

1. Caticha, A. Entropic Inference and the Foundations of Physics; USP Press: São Paulo, Brazil, 2012. Available online: http://www. albany.edu/physics / ACaticha-EIFP-book.pdf (accessed on 20 July 2021).

2. Amari, S.; Nagaoka, H. Methods of Information Geometry; Oxford University Press: Oxford, UK, 2000.

3. Cafaro, C. The Information Geometry of Chaos. Ph.D. Thesis, State University of New York, Albany, NY, USA, 2008.

4. Ali, S.A.; Cafaro, C.; Gassner, S.; Giffin, A. An information geometric perspective on the complexity of macroscopic predictions arising from incomplete information. Adv. Math. Phys. 2018, 2018, 2048521. [CrossRef]

5. Felice, D.; Cafaro, C.; Mancini, S. Information geometric methods for complexity. Chaos 2018, 28, 032101. [CrossRef]

6. Caticha, A. Entropic Dynamics. AIP Conf. Proc. 2002, 617, 302.

7. Cafaro, C.; Ali, S.A. Maximum caliber inference and the stochastic Ising model. Phys. Rev. 2016, E94, 052145. [CrossRef]

8. Ali, S.A.; Cafaro, C. Theoretical investigations of an information geometric approach to complexity. Rev. Math. Phys. 2017, 29, 1730002. [CrossRef]

9. Cafaro, C.; Ali, S.A. The spacetime algebra approach to massive classical electrodynamics with magnetic monopoles. Adv. Appl. Clifford Algebr. 2007, 17, 23. [CrossRef] 
10. Cafaro, C.; Giffin, A.; Ali, S.A.; Kim, D.-H. Reexamination of an information geometric construction of entropic indicators of complexity. Appl. Math. Comput. 2010, 217, 2944. [CrossRef]

11. Kittel, C. Elementary Statistical Physics; John Wiley \& Sons, Inc.: Hoboken, NJ, USA, 1958.

12. Ito, S.; Oizumi, M.; Amari, S. Unified framework for the entropy production and the stochastic interaction based on information geometry. Phys. Rev. Res. 2020, 2, 033048. [CrossRef]

13. Kaniadakis, G. Statistical mechanics in the context of special relativity. Phys. Rev. 2002, E66, 056125. [CrossRef]

14. Fisher, R.A. Theory of statistical estimation. Proc. Cambridge Philos. Soc. 1925, 122, 700. [CrossRef]

15. Rao, C.R. Information and accuracy attainable in the estimation of statistical parameters. Bull. Calcutta Math. Soc. 1945, 37, 81.

16. Cencov, N.N. Statistical decision rules and optimal inference. Transl. Math. Monogr. Amer. Math. Soc. 1981, 53.

17. Campbell, L.L. An extended Cencov characterization of the information metric. Proc. Am. Math. Soc. 1986, $98,135$.

18. Weinberg, S. Gravitation and Cosmology; John Wiley \& Sons, Inc.: Hoboken, NJ, USA, 1972.

19. Lee, J.M. Riemannian Manifolds: An Introduction to Curvature; Springer: Berlin, Germany, 1997.

20. Cafaro, C.; Ali, S.A. Jacobi fields on statistical manifolds of negative curvature. Physica 2007, D70, 234. [CrossRef]

21. Ohanian, H.C.; Ruffini, R. Gravitation and Spacetime; W. W. Norton \& Company: New York, NY, USA, 1994.

22. Carmo, M.P.D. Riemannian Geometry; Birkhauser: Basel, Switzerland, 1992.

23. Cafaro, C.; Ali, S.A.; Giffin, A. An application of reversible entropic dynamics on curved statistical manifolds. AIP Conf. Proc. 2006, 872, 243.

24. Cafaro, C. Information geometry and chaos on negatively curved statistical manifolds. AIP Conf. Proc. 2007, $954,175$.

25. Cafaro, C. Recent theoretical progress on an information geometrodynamical approach to chaos. AIP Conf. Proc. 2008, $1073,16$.

26. Ali, S.A.; Cafaro, C.; Giffin, A.; Kim, D.-H. Complexity characterization in a probabilistic approach to dynamical systems through information geometry and inductive inference. Phys. Scr. 2012, 85, 025009. [CrossRef]

27. Cafaro, C. Information geometric complexity of entropic motion on curved statistical manifolds. In Proceedings of the 12th Joint European Thermodynamics Conference, Brescia, Italy, 1-5 July 2013; Pilotelli, M., Beretta, G.P., Eds.; Cartolibreria Snoopy: Brescia, Italy, 2013; pp. 110-118.

28. Cafaro, C. Information-geometric indicators of chaos in Gaussian models on statistical manifolds of negative Ricci curvature. Int. J. Theor. Phys. 2008, 47, 2924. [CrossRef]

29. Ali, S.A.; Cafaro, C.; Kim, D.-H.; Mancini, S. The effect of microscopic correlations on the information geometric complexity of Gaussian statistical models. Physica 2010, A389, 3117. [CrossRef]

30. Caticha, A.; Cafaro, C. From information geometry to Newtonian dynamics. AIP Conf. Proc. 2007, $954,165$.

31. Zurek, W.H.; Paz, J.P. Decoherence, chaos, and the second law. Phys. Rev. Lett. 1994, 72, 2508. [CrossRef]

32. Zurek, W.H.; Paz, J.P. Quantum chaos: A decoherent definition. Physica 1995, D83, 300. [CrossRef]

33. Cafaro, C.; Ali, S.A. Geometrodynamics of information on curved statistical manifolds and its applications to chaos. EJTP 2008, $5,139$.

34. Cafaro, C. Works on an information geometrodynamical approach to chaos. Chaos Solitons Fractals 2009, 41, 886. [CrossRef]

35. Cafaro, C. Information geometry, inference methods and chaotic energy levels statistics. Mod. Phys. Lett. 2008, B22, 1879. [CrossRef]

36. Cafaro, C.; Ali, S.A. Can chaotic quantum energy levels statistics be characterized using information geometry and inference methods? Physica 2008, A387, 6876. [CrossRef]

37. Prosen, T.; Znidaric, M. Is the efficiency of classical simulations of quantum dynamics related to integrability? Phys. Rev. 2007, E75, 015202. [CrossRef]

38. Prosen, T.; Pizorn, I. Operator space entanglement entropy in transverse Ising chain. Phys. Rev. 2007, A76, 032316. [CrossRef]

39. Cafaro, C.; Mancini, S. On the complexity of statistical models admitting correlations. Phys. Scr. 2010, 82, 035007. [CrossRef]

40. Cafaro, C.; Mancini, S. Quantifying the complexity of geodesic paths on curved statistical manifolds through information geometric entropies and Jacobi fields. Phys. D Nonlinear Phenom. 2011, 240, 607. [CrossRef]

41. Kim, D.-H.; Ali, S.A.; Cafaro, C.; Mancini, S. An information geometric analysis of entangled continuous variable quantum systems. J. Phys. Conf. Ser. 2011, 306, 012063. [CrossRef]

42. Kim, D.-H.; Ali, S.A.; Cafaro, C.; Mancini, S. Information geometric modeling of scattering induced quantum entanglement. Phys. Lett. 2011, A375, 2868. [CrossRef]

43. Kim, D.-H.; Ali, S.A.; Cafaro, C.; Mancini, S. Information geometry of quantum entangled wave-packets. Physica 2012, A391, 4517. [CrossRef]

44. Cafaro, C.; Giffin, A.; Lupo, C.; Mancini, S. Insights into the softening of chaotic statistical models by quantum considerations. AIP Conf. Proc. 2012, 1443, 366.

45. Ali, S.A.; Cafaro, C.; Giffin, A.; Lupo, C.; Mancini, S. On a differential geometric viewpoint of Jaynes' MaxEnt method and its quantum extension. AIP Conf. Proc. 2012, 1443, 120.

46. Giffin, A.; Ali, S.A.; Cafaro, C. Local softening of chaotic statistical models with quantum consideration. AIP Conf. Proc. 2013, 1553, 238.

47. Cafaro, C.; Giffin, A.; Lupo, C.; Mancini, S. Softening the complexity of entropic motion on curved statistical manifolds. Open Syst. Inf. Dyn. 2012, 19, 1250001. [CrossRef] 
48. Giffin, A.; Ali, S.A.; Cafaro, C. Local softening of information geometric indicators of chaos in statistical modeling in the presence of quantum-like considerations. Entropy 2013, 15, 4622. [CrossRef]

49. Felice, D.; Cafaro, C.; Mancini, S. Information geometric complexity of a trivariate Gaussian statistical model. Entropy 2014, 16, 2944. [CrossRef]

50. Sadoc, J.F.; Mosseri, R. Geometrical Frustration; Cambridge University Press: Cambridge, UK, 2006.

51. Greven, A.; Keller, G.; Warnecke, G. Entropy; Princeton University Press: Princeton, NJ, USA, 2003.

52. Peres, A. Quantum Theory: Concepts and Methods; Kluwer Academic Publishers: London, UK, 1995

53. Peng, L.; Sun, H.; Xu, G. Information geometric characterization of the complexity of fractional Brownian motion. J. Math. Phys. 2012, 53, 123305. [CrossRef]

54. Peng, L.; Sun, H.; Sun, D.; Yi, J. The geometric structures and instability of entropic dynamical models. Adv. Math. 2011, 227, 459. [CrossRef]

55. Semarak, O.; Sukova, P. Free motion around black holes with discs or rings: between integrability and chaos-I. Mon. Not. R. Astron. Soc. 2010, 404, 545. [CrossRef]

56. Li, C.; Sun, H.; Zhang, S. Characterization of the complexity of an ED model via information geometry. Eur. Phys. J. Plus 2013, 128, 70. [CrossRef]

57. Cao, L.; Li, D.; Zhang, E.; Sun, H. A statistical cohomogeneity one metric on the upper plane with constant negative curvature. Adv. Math. Phys. 2014, 2014, 832683. [CrossRef]

58. Felice, D.; Mancini, S.; Pettini, M. Quantifying networks complexity from information geometry viewpoint. J. Math. Phys. 2014, 55, 043505. [CrossRef]

59. Abtahi, S.M.; Sadati, S.H.; Salarieh, H. Ricci-based chaos analysis for roto-translatory motion of a Kelvin-type gyrostat satellite. J.-Multi-Body Dyn. 2014, 228, 34. [CrossRef]

60. Mikes, J.; Stepanova, E. A five-dimensional Riemannian manifold with an irreducible SO(3)-structure as a model of abstract statistical manifold. Ann. Glob. Anal. Geom. 2014, 45, 111. [CrossRef]

61. Weis, S. Continuity of the maximum-entropy inference. Commun. Math. Phys. 2014, 330, 1263.

62. Li, C.; Peng, L.; Sun, H. Entropic dynamical models with unstable Jacobi fields. Rom. Journ. Phys. 2015, 60, 1249.

63. Itoh, M.; Satoh, H. Geometry of Fisher information metric and the barycenter map. Entropy 2015, 17, 1814. [CrossRef]

64. Franzosi, R.; Felice, D.; Mancini, S.; Pettini, M. A geometric entropy detecting the Erdös-Rényi phase transition. Eur. Phys. Lett. 2015, 111, 20001. [CrossRef]

65. Martins, A.C.R. Opinion particles: Classical physics and opinion dynamics. Phys. Lett. 2015, A379, 89. [CrossRef]

66. Muhammad, S.A.; Zhang, E.; Sun, H. Jacobi fields on the manifold of Freund. Ital. J. Pure Appl. Math. $2015,34,181$.

67. Felice, D.; Mancini, S. Gaussian network's dynamics reflected into geometric entropy. Entropy 2015, 17, 5660. [CrossRef]

68. Wen-Haw, C. A review of geometric mean of positive definite matrices. Br. J. Math. Comput. 2015, 5, 1.

69. Weis, S.; Knauf, A.; Ay, N.; Zhao, M.-J. Maximizing the divergence from a hierarchical model of quantum states. Open Syst. Inf. Dyn. 2015, 22, 1550006. [CrossRef]

70. Weis, S. Maximum-entropy inference and inverse continuity of the numerical range. Rep. Math. Phys. 2015, 77, 251. [CrossRef]

71. Shalymov, D.S.; Fradkov, A.L. Dynamics of non-stationary processes that follow the maximum of the Rényi entropy principle. Proc. R. Soc. 2016, A472, 20150324. [CrossRef] [PubMed]

72. Henry, G.; Rodriguez, D. On the instability of two entropic dynamical models. Chaos Solitons Fractals 2016, 91, 604. [CrossRef]

73. Gomez, I.S.; Portesi, M. Ergodic statistical models: Entropic dynamics and chaos. AIP Conf. Proc. 2017, $1853,100001$.

74. Gomez, I.S. Notions of the ergodic hierarchy for curved statistical manifolds. Physica 2017, A484, 117. [CrossRef]

75. Gassner, S.; Cafaro, C. Information geometric complexity of entropic motion on curved statistical manifolds under different metrizations of probability spaces. Int. J. Geom. Methods Mod. 2019, 16, 1950082. [CrossRef]

76. Gomez, I.S.; Portesi, M.; Borges, E.P. Universality classes for the Fisher metric derived from relative group entropy. Physica 2020, A547, 123827. [CrossRef]

77. Summers, R.L. Experiences in the Biocontinuum: A New Foundation for Living Systems; Cambridge Scholar Publishing: Cambridge, $\mathrm{UK}, 2020$.

78. Deshmukh, S.; Ishan, A.; Al-Shaik, S.B.; Özgür, C. A note on Killing calculus on Riemannian manifolds. Mathematics 2021, 9, 307. [CrossRef] 